\title{
MS03-P06 | Crystal Production And Structure Solution Thanks To The Nucleating And Phasing Agent, Crystallophore.
}

Girard, Eric (Institute of Structural Biology IBS, Grenoble, FRA); Engilberge, Sylvain (Institute for Structural Biology (IBS), Grenoble, FRA); Riobé, François (École Normale Supérieure, Lyon, FRA); Santoni, Gianluca (European Synchrotron Radiation Facility, Grenoble, FRA); Wagner, Tristan (Max-Planck-Institute for Marine Microbiology, Bremen, GER); Maury, Olivier (École Normale Supérieure, Lyon, FRA)

Obtaining crystals and solving the phase problem remain major hurdles encountered by bio-crystallographers in their race to get new high-quality structures.

The crystallophore, $\mathrm{Tb}-\mathrm{X}_{\mathrm{O}} 4$, is a lanthanide complex formed from a molecular cage chelating a terbium atom [2]. We will present the results obtained on about fifteen proteins which show that $\mathrm{X}_{04} 4$ induces unique crystallization conditions and promotes new crystal packing showing that Tb-Хo4 acts an efficient nucleating agent. The crystalline forms promoted by the crystallophore bypass crystal defects often encountered by crystallographers such as low-resolution diffracting samples or crystals with twinning.

We will also present few examples of $\mathrm{Tb}$-Xo4 phasing properties [1,2,4], showing, in particular that the crystallophore is compatible with serial crystallography approaches.

Finally, the versatility of the interactions between $\mathrm{X}_{04}$ and the surface of proteins explains its exceptional properties [3] making this molecule a unique tool for simultaneously solving the two major locks of biocrystallography.

[1] Bernhardsgrütter, I., et al. (2018). Nature Chemical Biology. 14, 1127-1132.

[2] Engilberge, S., et al. (2017). Chem. Sci. 8, 5909-5917.

[3] Engilberge, S., et al. (2018). Chemistry. 24, 9739-9746.

[4] Vögeli, B., et al. (2018). Proceedings of the National Academy of Sciences. 115, 3380-3385. 